\title{
CriptoData: Ensino de Criptografia via Computação Desplugada
}

\author{
Débora Juliane Guerra Marques da Silva ${ }^{1}$, Graziela Ferreira Guarda ${ }^{1}$
}

${ }^{1}$ Departamento de Computação - Universidade Católica de Brasília (UCB) Campus I - QS 07 - Lote 01 - EPCT - Águas Claras - Brasília - DF CEP: 71966-700

deborajuliane@gmail.com, grazielafguarda@gmail.com

\begin{abstract}
The Logicamente project was created to develop an educational methodology with learning objects using creative learning, computational thinking, maker culture and unplugged computing as pillars for Basic Education. Among the planned activities, it was idealized to perform playful activities with the purpose of fixing previously worked content or introducing new knowledge. In this context, CriptoData emerged which explores cryptography and programming logic based on the educational digital game Run Marco. The present article is to report the experiences about the application of the game in question that aimed to address issues related to Information Security.
\end{abstract}

Resumo. O projeto Logicamente foi criado para desenvolver uma metodologia educacional com objetos de aprendizagem utilizando a aprendizagem criativa, pensamento computacional, cultura maker e computação desplugada como pilares para a Educação Básica. Dentre as atividades previstas, se idealizou a realização de atividades lúdicas com o propósito de fixar conteúdos já trabalhados ou introduzir novos saberes. Neste contexto, surgiu o CriptoData que explora a criptografia e lógica de programação baseado no jogo digital educacional Run Marco. O presente artigo consiste em relatar as experiências acerca da aplicação do jogo em questão que teve por objetivo abordar temas relacionados à Segurança da Informação.

\section{Introdução}

O ensino de conteúdos de Computação para a Educação Básica é essencial na atualidade. Através da inclusão desses novos saberes, os estudantes tendem a compreender de forma mais completa o mundo e, consequentemente, poderão se tornar capazes de ter mais autonomia, flexibilidade, resiliência, pró-atividade e criatividade que são competências necessárias no mundo contemporâneo.

Neste sentido, é fundamental que as habilidades do Pensamento Computacional sejam trabalhadas na Educação Básica para que seja possível aos estudantes no futuro, se tornarem capazes de construir modelos mentais para as abstrações computacionais, que serão formalizadas com o uso de linguagens de programação. [SBC 2017].

O Pensamento Computacional (PC) pode ser compreendido como um instrumento interventor que auxilia na resolução de problemas e compreensão do comportamento humano, baseado nos conceitos fundamentais da Ciência da Computação, o PC dispõe de ferramentas mentais que refletem a amplitude do campo das Ciências Exatas [Wing 2006]. Já a Computação Desplugada (CD), pode ser 
compreendida como um método de ensino com o objetivo de explicar os fundamentos da Computação sem a necessidade de um computador, esse método foi criado com o propósito de possibilitar que indivíduos que não possuem acesso ao computador tenham a oportunidade de entender o seu funcionamento e seus fundamentos [Bell et al., 2009].

Dentre as diversas possibilidades de conteúdos de Computação a serem ministrados para a Educação Básica, o presente estudo elegeu o ensino da criptografia. A criptografia pode ser compreendida como um conjunto de métodos e técnicas para cifrar ou codificar informações legíveis por meio de um algoritmo, convertendo um texto original em um texto ilegível, sendo possível mediante o processo inverso a recuperação das informações originais [Guarda et at. 2018]. Essa escolha foi feita considerando a importância do diálogo acerca do tema da Segurança da Informação com os estudantes ressaltando os riscos e cuidados inerentes a temática.

A atividade proposta se refere a um conjunto de ações do Projeto Logicamente que é uma atividade de pesquisa cujo enfoque é desenvolver uma metodologia educacional e objetos de aprendizagem utilizando as premissas das aprendizagens criativas, pensamento computacional, cultura maker e computação desplugada como pilares. Neste sentido, foi elaborada a atividade denominada "CriptoData: Ensino de Criptografia via Computação Desplugada" cujo propósito foi trabalhar os conceitos de criptografia usando comandos de lógica de programação inspirado no jogo digital educacional Run Marco [Run Marco 2018] com o Ensino Fundamental - Anos Finais.

O estudo está dividido da seguinte maneira: a seguir, na Seção 2, é apresentada uma explicação sobre a atividade e a metodologia utilizada, bem como, sua organização estrutural. Os resultados parciais são descritos na Seção 3. Por fim, os objetivos e metas desta experiência serão destacados na Seção 4, de forma a concluir o propósito da atividade diante dos resultados já obtidos.

\section{Proposta e Metodologia}

A ideia da atividade surgiu oriunda do conjunto de atividades abordadas pelo projeto Logicamente como ferramenta para desenvolvimento do aprendizado. O público-alvo foram duas turmas de uma escola particular do Distrito Federal. Turma 1: Turma iniciante no projeto com estudantes do $7^{\circ}$ e $8^{\circ}$ anos e Turma 2: Turma avançada no projeto com estudantes de $6^{\circ}$ e $7^{\circ}$ anos que estão participando do projeto em seu segundo ano consecutivo. No ano de 2018, o projeto Logicamente atendeu diferentes turmas do Ensino Fundamental - Anos Finais onde cada uma delas teve um encontro semanal com duração de 1 hora e 40 minutos no contra turno.

A atividade teve por objetivo ensinar técnica de criptografia com uso de lógica de programação inspirado no jogo digital educacional Run Marco objetivando o desenvolvimento das habilidades do PC como: Abstração - capacidade de filtrar informações essenciais e descartar as informações desnecessárias em um determinado contexto; Decomposição - dividir um problema grande em partes menores, facilitando sua solução; Coleta de Dados - localizar dados necessários para resolver um problema; e Construção de Algoritmo - sequências de passos ordenados para se atingir um determinado objetivo [Pessoa et al, 2017].

Preliminarmente, para fins de melhor aproveitamento da atividade, nas duas semanas anteriores a aplicação da mesma, foram realizadas aulas preparatórias com 
VIII Congresso Brasileiro de Informática na Educação (CBIE 2019)

Anais dos Workshops do VIII Congresso Brasileiro de Informática na Educação (WCBIE 2019)

intuito de minimizar eventuais dúvidas relacionadas aos conteúdos que seriam abordados. Nesses encontros, as turmas tiveram a oportunidade de jogar a versão digital do jogo Run Marco para que os fundamentos de programação já fossem trabalhados com os estudantes.

\subsection{A dinâmica da atividade}

A técnica de criptografia escolhida para implementação da atividade CriptoData foi a "Cifra de César". Essa técnica se baseia na utilização das letras do alfabeto, as substituindo (no caso da atividade proposta) por números naturais (sem casas decimais). Essa cifra está classificada como monoalfabética, caracterizada pela substituição de uma letra por outra ou por um símbolo para criptografar uma mensagem. Essa técnica é de fácil compreensão e já havia sido utilizada no contexto da Educação Básica como mostra o estudo de [Groenwald et al. 2010]. Neste sentido, a Figura 1 abaixo ilustra a Codificação Cifra de César adotada na atividade. O esquema foi elaborado utilizando a seguinte regra: as letras do alfabeto foram substituídas por números seguindo esse esquema numérico sequencial: $\mathrm{A}=1 ; \mathrm{B}=2 ; \mathrm{C}=3 ; \mathrm{D}=4$ e assim sucessivamente (incluindo os caracteres $\mathrm{K}, \mathrm{Y}, \mathrm{W}$ e \#).

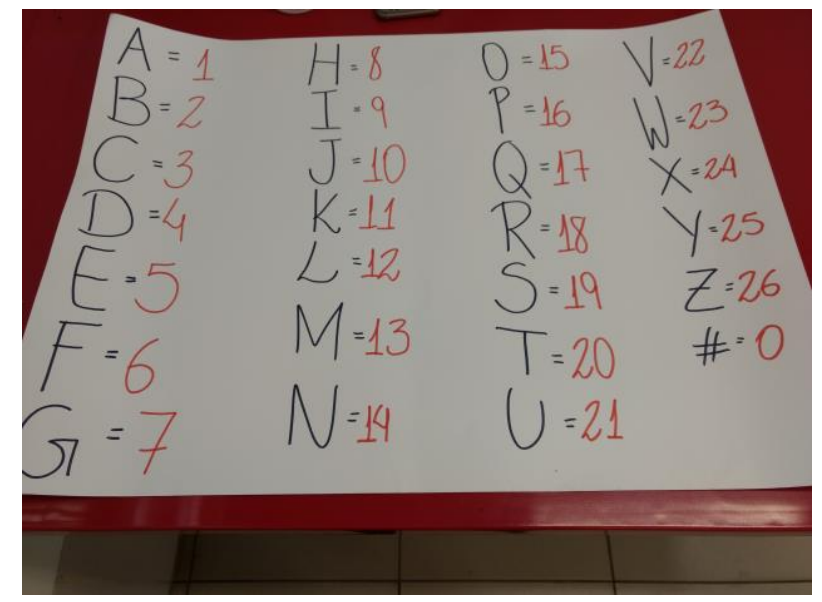

Figura 1. Codificação Cifra de César adotada

Para realização da dinâmica, os estudantes foram divididos em grupos de quatro a cinco estudantes que primeiramente deveriam escolher as fases do jogo Run Marco que iriam resolver para descobrir a chave codificadora/decodificadora das mensagens criptografadas. Essas escolhas das fases foram realizadas aleatoriamente pelos grupos e esse processo se repetiu três vezes sendo: uma fase (principal) para obter o acesso à chave e as outras duas fases para obter um bônus de pontuação se conseguissem resolver a fase principal em até 2 minutos (opcional). A atividade disponibilizou 30 fases do jogo Run Marco impressas em papel A4 no total para escolha dos grupos.

As resoluções das fases escolhidas seriam utilizadas pelos grupos para obterem a chave de acesso. Para solucionar as fases, era necessário fazer o personagem do jogo chegar na "estrelinha". Para fins de definição da chave, foi contada a quantidade de instruções algorítmicas que foram necessárias para o personagem atingir o objetivo conforme mostrado na Figura 2 - um exemplo de uma das fases resolvida por um dos grupos. 
VIII Congresso Brasileiro de Informática na Educação (CBIE 2019)

Anais dos Workshops do VIII Congresso Brasileiro de Informática na Educação (WCBIE 2019)

No exemplo citado, o boneco executou 14 instruções algorítmicas para chegar a "estrelinha", e, deste modo, 14 era o valor da chave. Após os grupos resolverem as fases, os gabaritos foram entregues pelos grupos para os monitores da atividade que fizeram a conferência para verificar se o gabarito estava correto, estando correto eles recebiam a chave codificadora/decodificadora - essa chave é necessária para decifrar o conteúdo das mensagens criptografadas.

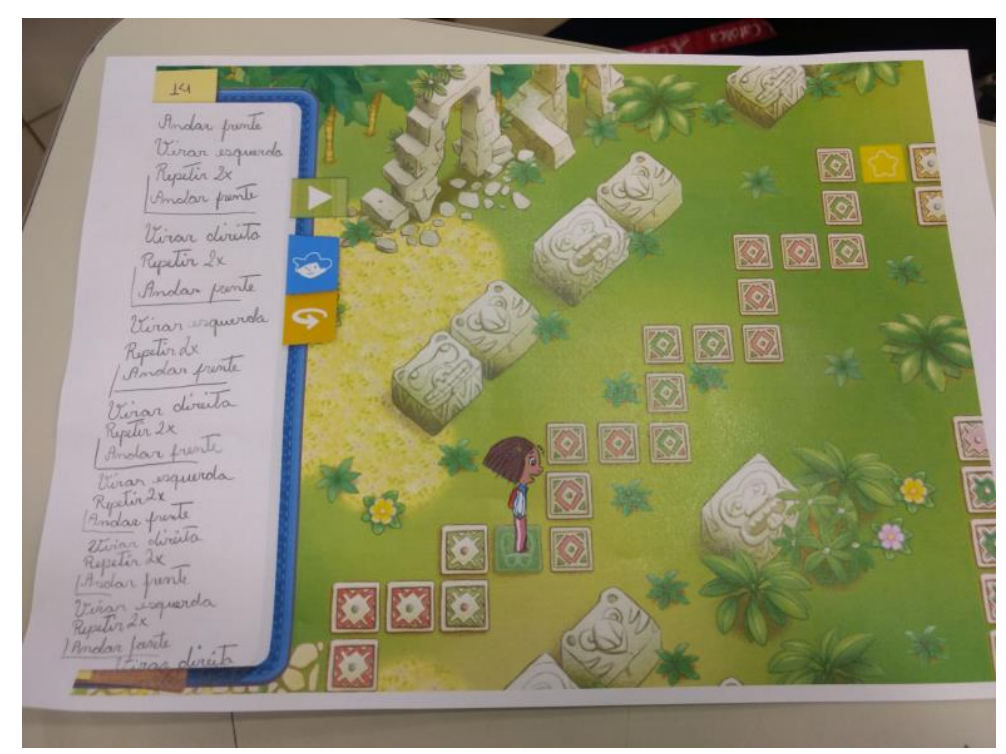

Figura 2. Fase do Run Marco resolvida

Em relação às regras do jogo. As mensagens ficaram dispostas no quadro já criptografadas para que os grupos pudesses traduzí-las (Figura 3) de acordo com a chave. A regra usada para criptografar as mensagens foi somar os números ao valor da chave. Exemplo: palavra FIM: 69 e 13 (ver Figura 1), se a chave fosse 19 após a criptografia a mensagem ficaria 2528 32. Já para decifrar a mensagem (descriptografar), a regra era inversa, deveria ser feita a subtração em relação ao valor da chave. Na Figura 4 é demonstrado uma das mensagens decifradas por um dos grupos cuja chave era 19. Deste modo, 25 que era a primeira letra da mensagem foi subtraído por 19 que ficou 6, 6 equivale a letra $\mathrm{F}$ no mapa da Cifra de César criado para o jogo.

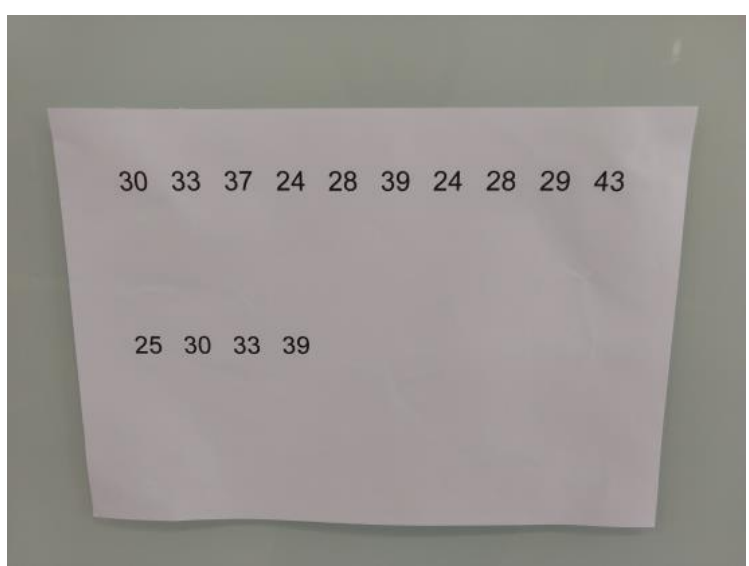

Figura 3. Exemplo de mensagem criptografada

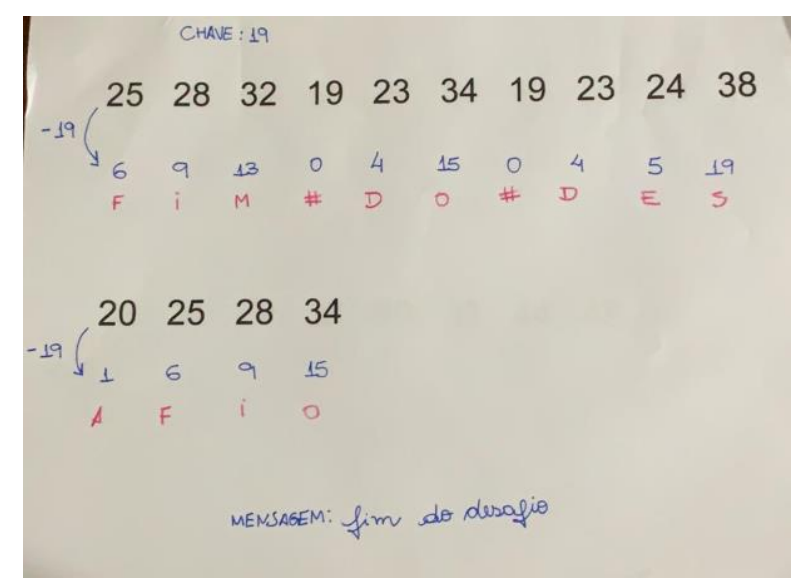

Figura 4. Exemplo de mensagem descriptografada 
VIII Congresso Brasileiro de Informática na Educação (CBIE 2019)

Anais dos Workshops do VIII Congresso Brasileiro de Informática na Educação (WCBIE 2019)

Em relação as condições de jogabilidade. Os grupos começaram o jogo com 100 pontos. O tempo gasto pelas equipes foram cronometrados. Caso as equipes optassem pela solução de fases bônus, as mesmas não poderiam ultrapassar 5 minutos para construção. O tempo máximo para finalização do jogo foi de 1 hora e 40 minutos período dos encontros semanais do Projeto Logicamente. A pontuação final foi calculada da seguinte forma: 100 - tempo do desafio (cronometrado por equipe) + bônus - penalidades. A equipe vencedora foi a equipe que no final computasse o maior número de pontos. Em relação a pontuação, foram definidos os critérios abaixo:

-Barulho demais -10

-Membro do grupo que não participasse -10

- Trabalho em equipe +10

-Fase Bônus +10

•Usar "Se.. em Seguida" +10

•Usar "Repetir... vezes" + 10

-Usar "Repetir.. enquanto caminho de" +15

Os movimentos permitidos no jogo estão demonstrados na Figura 5 Movimentos permitidos Run Marco.

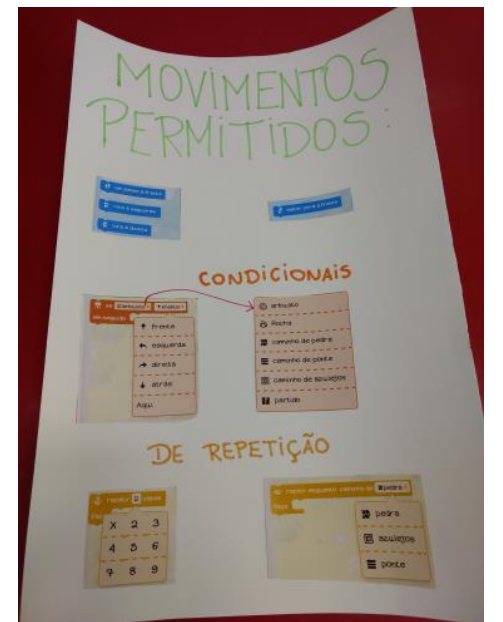

Figura 5. Movimentos permitidos Run Marco

\section{Resultados e Discussões}

O CriptoData foi jogado por um total de 40 (quarenta) estudantes que foram divididos em 9 (nove) equipes de duas turmas diferentes do projeto Logicamente.

A turma 1: Turma iniciante no projeto com estudantes de $7^{\circ}$ e $8^{\circ}$ anos do Ensino Fundamental (Anos Finais). Foram montados 3 grupos de 4 estudantes e 1 grupo com 5 estudantes. Totalizando 17 participantes. 
VIII Congresso Brasileiro de Informática na Educação (CBIE 2019)

Anais dos Workshops do VIII Congresso Brasileiro de Informática na Educação (WCBIE 2019)

A turma 2: Turma avançada no projeto com estudantes de $6^{\circ}$ e $7^{\circ}$ anos do Ensino Fundamental (Anos Finais). Foram montados 2 grupos de 4 estudantes e 3 grupos com 5 estudantes. Totalizando 23 participantes. Os resultados de ambos os grupos foram compilados nas Tabelas 1 e 2 a seguir:

Tabela 1. Resultados da Turma 1

\begin{tabular}{|c|c|c|c|c|c|c|}
\hline & $\begin{array}{l}\text { Tempo } \\
\text { gasto: }\end{array}$ & Penalidades: & $\begin{array}{l}\text { Quantidade } \\
\text { de Fases } \\
\text { Bônus: }\end{array}$ & $\begin{array}{l}\text { Pontuação } \\
\text { por Trabalho } \\
\text { em Equipe: }\end{array}$ & $\begin{array}{l}\sum \text { Uso das } \\
\text { estruturas } \\
\text { (condicionais } \\
\text { ou repetição): }\end{array}$ & $\begin{array}{l}\text { Pontuação } \\
\text { Final: }\end{array}$ \\
\hline G1 & 30 & $\begin{array}{l}\text {-10 } \\
\text { estudante } \\
\text { não } \\
\text { participou) }\end{array}$ & $1=+10$ & +10 & +40 & $\begin{array}{l}100-30+ \\
10+10+ \\
40=130\end{array}$ \\
\hline G2 & 35 & NA & $2=+20$ & +10 & +70 & $\begin{array}{l}100-35+ \\
20+10+ \\
70=165\end{array}$ \\
\hline G3 & 18 & $\mathrm{NA}$ & $2=+20$ & +10 & +75 & $\begin{array}{l}100-18+ \\
20+10+ \\
75=187\end{array}$ \\
\hline G4 & 20 & $\mathrm{NA}$ & $2=+20$ & +10 & +40 & $\begin{array}{l}100-20+ \\
20+10+ \\
40=140\end{array}$ \\
\hline Med & 25,75 & & & & & 155,50 \\
\hline
\end{tabular}

Tabela 2. Resultados da Turma 2

\begin{tabular}{|c|c|c|c|c|c|c|}
\hline & $\begin{array}{l}\text { Tempo } \\
\text { gasto: }\end{array}$ & Penalidades: & $\begin{array}{l}\text { Quantidade } \\
\text { de Fases } \\
\text { Bônus: }\end{array}$ & $\begin{array}{l}\text { Pontuação } \\
\text { por } \\
\text { Trabalho } \\
\text { em Equipe: }\end{array}$ & $\begin{array}{l}\sum \text { Uso das } \\
\text { estruturas } \\
\text { (condicionais } \\
\text { ou } \\
\text { repetição): }\end{array}$ & $\begin{array}{l}\text { Pontuação } \\
\text { Final: }\end{array}$ \\
\hline G1 & 44 & NA & $2=+20$ & +10 & +135 & $\begin{array}{l}100-44+20+ \\
10+135=221\end{array}$ \\
\hline G2 & 47 & NA & $2=+20$ & +10 & +70 & $\begin{array}{l}100-47+20+ \\
80=153\end{array}$ \\
\hline G3 & 25 & NA & $1=+10$ & +10 & +60 & $\begin{array}{l}100-25+10+ \\
10+60=155\end{array}$ \\
\hline G4 & 25 & NA & $2=+20$ & +10 & +120 & $\begin{array}{l}100-25+20+ \\
10+120=225\end{array}$ \\
\hline G5 & 15 & $\mathrm{NA}$ & $2=+20$ & +10 & +180 & $\begin{array}{l}100-15+20+ \\
10+180=295\end{array}$ \\
\hline Med & 31,20 & & & & & 209,80 \\
\hline
\end{tabular}

O valor 'NA' contido nas colunas 'Penalidades' significa 'Não se Aplica', ou seja, a equipe não foi penalizada na pontuação. Conforme demonstrado na Tabela 1 , o grupo vencedor da Turma 1 foi o $\mathbf{G 3}$ com 187 pontos, já na Tabela 2 foi demonstrado que o grupo vencedor foi o $\mathbf{G 5}$ com 295 pontos.

Os dados demonstrados nas tabelas acima demonstraram ainda que a Turma 2 Avançada do Projeto Logicamente gastou em média mais tempo para finalização do jogo em relação ao Grupo 1 (31,20 x 25,75 minutos), o que demonstra "maturidade" da 
VIII Congresso Brasileiro de Informática na Educação (CBIE 2019)

Anais dos Workshops do VIII Congresso Brasileiro de Informática na Educação (WCBIE 2019)

turma em perceber que as análises tanto para desenvolver as fases do Run Marco quanto para criptografar e descriptografar as mensagens necessitavam de concentração dos estudantes e conferências para evitar erros nos cálculos. Essa consciência por parte dos estudantes ocasionou uma pontuação muito maior quando comparado a pontuação da equipe campeã da Turma 2 em relação a pontuação da equipe campeã da Turma 1 . Neste caso, a Turma 2 obteve um rendimento $47 \%$ melhor que a equipe da Turma 1.

As pontuações finais no geral da Turma 2 também foram superiores as alcançadas pela Turma 1, o que era esperado considerando que a Turma 2 está participando das atividades do Projeto Logicamente há dois anos enquanto que a Turma 1 está no seu primeiro ano. Esse dado indica a melhora de rendimento da turma com o passar do tempo e a exposição dos variados conteúdos de Computação que estão sendo ministrado pela equipe do projeto.

Outro aspecto interessante demonstrado nas tabelas é a quantidade de pontuações em relação ao uso das estruturas (sequenciais e de repetição) utilizadas pela Turma 2 (Figura 4), essa turma usou uma quantidade maior de estruturas mais complexas quando comparada com a Turma 1. Em relação as fases bônus, as tabelas demonstraram que ambas as turmas fizeram as fases bônus que era opcional, esse dado indica que o jogo foi bem aceito pelas equipes e quanto ao trabalho ter sido desenvolvido em equipes, todas as equipes trabalharam bem essa divisão de tarefas.

Adicionalmente, foi aplicado um questionário avaliativo para fins de coleta de dados em relação a temática proposta que foi debater a importância da Segurança da Informação. As perguntas realizadas nos questionários estão dispostas abaixo, seguidas das opções de resposta. O questionário foi aplicado para todos os quarenta estudantes que jogaram o CriptoData.

P1: Você gostou do jogo? Sim, Não.

P2: Como você classifica o nível de dificuldade do jogo? Fácil, Médio, Difícil.

P3: Você teve dificuldade com o trabalho em equipe? Sim, Não.

P4: Você contribuiu com o trabalho em equipe? Sim, Não, Não sei.

P5: No geral, o que você percebeu da atividade? Aberta.

P6: O que você entende por segurança de dados? Aberta.

P7: Para você, qual a importância da criptografia na segurança de dados? Aberta.

P8: Você utiliza redes sociais? Sim, Não.

P9: Seus pais têm algum controle / acompanhamento das suas contas nas redes sociais? Sim, Não, Não sei, Não sei aplica (para os que não tem redes sociais).

P10: Dentro da rede social você privatiza seu perfil? Sim, Não, Não se aplica (para os que não tem redes sociais).

P11: Você acha que suas fotos e publicações são seguras? Sim, Não, Não sei.

P12: Quais tipos de cuidados você toma para evitar que aconteça o vazamento de informações na sua rede social ou em seus dispositivos? Aberta.

P13: Você já teve alguma conta hackeada? Sim, Não, Não sei.

P14: Você conhece alguém que teve a conta hackeada ou teve informações vazadas? Sim, Não.

P15: Você consegue perceber a relação entre o lúdico com o tema segurança da informação? Sim, Não.

As respostas do questionário acima foram compiladas nas Tabelas 3 e 4 respectivamente, como segue: 
VIII Congresso Brasileiro de Informática na Educação (CBIE 2019)

Anais dos Workshops do VIII Congresso Brasileiro de Informática na Educação (WCBIE 2019)

Tabela 3. Questionário Avaliativo - Turma 1

\begin{tabular}{|l|l|}
\hline & Respostas: \\
\hline P1: & 16 Sim e 1 Não. \\
\hline P2: & 8 Fácil, 8 Médio e 1 Difícil. \\
\hline P3: & 17 Não. \\
\hline P4: & 11 Sim, 2 Não e 4 Não sei. \\
\hline P5: & $\begin{array}{l}\text { Pressão em relação ao tempo, diversão, necessidade de articulação para resolução de } \\
\text { problemas, necessidade de trabalho em equipe, Percepção de existir diferentes formas de } \\
\text { resolver o enigma do Run Marco, Percepção da otimização na construção dos algoritmos } \\
\text { usando estruturas (condicionais e repetição), melhora na capacidade de resolução de } \\
\text { problemas com o trabalho em equipe, importância do conhecimento sobre o tema. }\end{array}$ \\
\hline P6: & $\begin{array}{l}\text { Que os dados ficam mais seguros e protegidos, percebeu-se a necessidade de maior } \\
\text { proteção das senhas de acesso, percebeu-se a necessidade dos cuidados básicos quanto a } \\
\text { segurança das informações no geral, percebeu-se que é algo importante, percebeu-se a } \\
\text { importância de se ter um backup dos dados. }\end{array}$ \\
\hline P7: & $\begin{array}{l}\text { Forma de proteção dos dados, possibilidade de comunicação restrita entre pessoas ou } \\
\text { grupos, possibilidade de esconder segredos. }\end{array}$ \\
\hline P8: & 14 Sim e 3 Não. \\
\hline P9: & 7 Sim, 7 Não Sei e 3 Não se aplica. \\
\hline P10: & 8 Sim, 6 Não e 5 Não se aplica. \\
\hline P11: & 10 Sim, 1 Não e 6 Não sei. \\
\hline P12: & $\begin{array}{l}\text { Sigilo quanto ao uso das senhas e não uso de senhas fáceis, Preocupação com os } \\
\text { conteúdos postados em redes sociais, Configuração de restrição nos perfis em redes } \\
\text { sociais. }\end{array}$ \\
\hline P13: & 1 Sim, 11 Não e 5 Não sei. \\
\hline P14: & 9 Sim e 8 Não. \\
\hline P15: & 10 Sim e 7 Não. \\
\hline
\end{tabular}

Tabela 4. Questionário Avaliativo - Turma 2

\begin{tabular}{|l|l|}
\hline & Respostas: \\
\hline P1: & 20 Sim e 3 Não. \\
\hline P2: & 13 Fácil, 10 Médio. \\
\hline P3: & 2 Sim e 21 Não. \\
\hline P4: & 21 Sim e 2 Não sei. \\
\hline P5: & $\begin{array}{l}\text { Importância do trabalho em equipe e melhora de rendimento, a percepção de que nem } \\
\text { sempre a solução mais fácil é a melhor, necessidade de calma e estratégia na resolução de } \\
\text { problemas, percepção da importância do tema trabalhado, percepção de proximidade com } \\
\text { o mundo real e importância do uso da lógica de programação em questões do mundo real, } \\
\text { percepção da fragilidade dos dados, riqueza no debate do trabalho em equipe, percepção } \\
\text { do uso da criptografia em questões pessoais sem conhecimento do tema, percepção da } \\
\text { ligação entre o tema criptografia e senhas, um caso isolado de relato de dificuldade de } \\
\text { trabalho em equipe - divergência de opiniões. }\end{array}$ \\
\hline P6: & $\begin{array}{l}\text { Percebeu-se a necessidade dos cuidados básicos quanto a segurança das informações no } \\
\text { geral, necessidade de maiores cuidados com a exposição de informaçães e dados na } \\
\text { internet, integração com a criptografia, que os dados ficam mais seguros e protegidos, } \\
\text { percebeu-se a necessidade de maior proteção das senhas de acesso, percebeu-se a } \\
\text { importância da não comunicação com estranhos (virtualmente). }\end{array}$ \\
\hline P7: & $\begin{array}{l}\text { Possibilidade de comunicação restrita entre pessoas ou grupos, possibilidade de esconder } \\
\text { segredos, forma de proteção dos dados, recurso de apoio a segurança das informações. }\end{array}$ \\
\hline P8: & 18 Sim e 5 Não. \\
\hline
\end{tabular}


VIII Congresso Brasileiro de Informática na Educação (CBIE 2019)

Anais dos Workshops do VIII Congresso Brasileiro de Informática na Educação (WCBIE 2019)

\begin{tabular}{|l|l|}
\hline P9: & 9 Sim, 6 Não, 3 Não Sei e 5 Não se aplica. \\
\hline P10: & 12 Sim, 6 Não e 5 Não se aplica. \\
\hline P11: & 12 Sim, 4 Não, 7 Não sei. \\
\hline P12: & $\begin{array}{l}\text { Cuidados em não deixar senhas em aparelhos não pessoais, preocupação com os } \\
\text { conteúdos postados em redes sociais, mudanças de senhas periodicamente, sigilo quanto } \\
\text { ao uso das senhas e não uso de senhas fáceis, necessidade de avaliação de sites seguros. }\end{array}$ \\
\hline P13: & 2 Sim e 21 Não. \\
\hline P14: & 14 Sim e 9 Não. \\
\hline P15: & 18 Sim e 5 Não. \\
\hline
\end{tabular}

Os dados contidos nas Tabelas 3 e 4 mostraram que grande parte dos estudantes gostaram da atividade, que a consideraram de nível médio de complexidade e que no geral, a temática da Segurança da Informação foi tratada e discutida deixando pontos de reflexão para todos. Por fim, em relação as habilidades do PC trabalhadas no jogo foram: Abstração, Coleta de Dados, Análise de Dados, Decomposição, Algoritmos e Procedimentos e Paralelização.

\section{Conclusões}

O projeto Logicamente tem como finalidade motivar crianças e adolescentes a aprenderem conteúdos de Computação que envolvem programação, lógica e assuntos relacionados ao PC de maneira criativa, que são habilidades essenciais para a vida de qualquer indivíduo. Se estima com a inserção o PC no âmbito da Educação Básica, oportunizar a formação de habilidades e competências computacionais, apoiando a ciência e suas áreas de conhecimento. Essas habilidades e competências potencializam a capacidade de resolver problemas.

O jogo teve por objetivo ensinar criptografia trabalhando conjuntamente lógica de programação em consonância com as habilidades do PC. As crianças, hoje, já nascem imersas em um mundo digital, mas, ao contrário do que se possa imaginar, elas não conhecem o funcionamento desse mundo, apenas utilizam suas ferramentas passivamente. A opção pela inclusão do tema criptografia foi de grande valia para que pudesse ser abordado com o público-alvo posteriormente, o tema segurança da informação, que envolve questões importantes sobre os riscos que somos expostos ao utilizar as tecnologias, os quais se pode destacar: casos de violação de contas bancárias, acesso a informações sigilosas, invasão e destruição de sistemas, entre outros.

De acordo com os resultados expostos na Seção 3 do presente estudo, o jogo foi considerado uma iniciativa interessante, que despertou curiosidade pelo tema abordado, interesse em pesquisas e favoreceu a compreensão dos assuntos relacionados reforçando os conteúdos trabalhados pelo projeto Logicamente. Adicionalmente, o uso da computação desplugada foi uma abordagem interessante se considerarmos sua utilização em espaços com pouca ou nenhuma infraestrutura de tecnologia computacional. Realidade essa, bastante presente no cenário da Educação Básica pública brasileira. Além disso, o uso de materiais de baixo custo para criação dos objetos do jogo, o torna um objeto de aprendizagem acessível para implementação em qualquer escola. 
VIII Congresso Brasileiro de Informática na Educação (CBIE 2019)

Anais dos Workshops do VIII Congresso Brasileiro de Informática na Educação (WCBIE 2019)

\section{Referências}

Bell, T., Alexander, J., Freeman, I., Grimley, M. (2009). Computer Science Unplugged: School students doing real computing without computers. The New Zealand Journal of Applied Computing and Information Technology, 13(1):20-29.

Groenwald, Cláudia; Olgin, Clarissa. Códigos e Senhas: Sequencia Didática com o tema Criptografia no Ensino Fundamental. X Encontro Nacional de Educação Matemática, 2010. Salvador. Anais http://www.lematec.net.br/CDS/ENEM10/artigos/CC/T17_CC555.pdf

Guarda, Graziela; Silva, Débora; Goulart, Ione. CriptoLab: Um game baseado em Computação Desplugada e Criptografia. In: Workshop sobre Educação em Computação (WEI), XXVI, 2018, Natal. Anais: Sociedade Brasileira de Computação - SBC, 2018. p. 49 - 59.

Pessoa, F. I. R; Araújo, A. S. O.; Andrade W. L.; Guerrero, D. D. S (2017) "T-mind: um Aplicativo Gamificado para Estímulo ao Desenvolvimento de Habilidades do Pensamento Computacional". In: Simpósio Brasileiro de Informática na Educação (SBIE), XXIII, 2017, Recife. Anais do SBIE. DOI: 10.5753/cbie.sbie.2017.645.

Run Marco. Jogo Digital Educacional - All Can Code. (2018, março). Disponível em https://www.brainpop.com/games/runmarco/

Sociedade Brasileira de Computação (2017) Diretrizes para Ensino de Computação na Educação Básica. http://www.sbc.org.br/documentos-da-sbc/send/131-curriculos-dereferencia/1177-diretrizes-para-ensino-de-computacao-na-educacao-basica, Agosto.

Wing, Jeannette M. Computational thinking. Communications Of the Acm, [s.1.], v. 49, n. 3, p.33-35, 1 mar. 2006. Association for Computing Machinery (ACM). http://dx.doi.org/10.1145/1118178.1118215. 